Portland State University

PDXScholar

$2-1-2008$

\title{
Measurements of dark current in a CCD imager during light exposures
}

\author{
Ralf Widenhorn \\ Portland State University \\ Ines Hartwig \\ Justin Charles Dunlap \\ Portland State University \\ Erik Bodegom \\ Portland State University
}

Follow this and additional works at: https://pdxscholar.library.pdx.edu/phy_fac

Part of the Physics Commons

Let us know how access to this document benefits you.

\section{Citation Details}

Ralf Widenhorn; Ines Hartwig; Justin C. Dunlap and Erik Bodegom, "Measurements of dark current in a CCD imager during light exposures," Proc. of SPIE 6816, Sensors, Cameras, and Systems for Industrial/ Scientific Applications IX, 68160B Morley M. Blouke; Erik Bodegom, Editors (Feb. 29, 2008); DOI: 10.1117/ 12.769082

This Article is brought to you for free and open access. It has been accepted for inclusion in Physics Faculty Publications and Presentations by an authorized administrator of PDXScholar. Please contact us if we can make this document more accessible: pdxscholar@pdx.edu. 


\title{
Measurements of dark current in a CCD imager during light exposures
}

\author{
Ralf Widenhorn, ${ }^{\mathrm{a}, \mathrm{b}}$ Ines Hartwig, ${ }^{\mathrm{a}}$ Justin C. Dunlap, ${ }^{\mathrm{a}}$ and Erik Bodegom ${ }^{\mathrm{a}, \mathrm{b}}$ \\ ${ }^{a}$ Portland State, Portland, OR 97207, ${ }^{b}$ Digital Clarity Consultants, Lyons, OR 97328,
}

\begin{abstract}
Thermal excitation of electrons is a major source of noise in Charge-Coupled Device (CCD) imagers. Those electrons are generated even in the absence of light, hence the name dark current. Dark current is particularly important for long exposure times and elevated temperatures. The standard procedure to correct for dark current is to take several pictures under the same condition as the real image, except with the shutter closed. The resulting dark frame is later subtracted from the exposed image. We address the question of whether the dark current produced in an image taken with a closed shutter is identical to the dark current produced in an exposure in the presence of light. In our investigation, we illuminated two different CCD chips to different intensities of light and measured the dark current generation. A surprising conclusion of this study is that some pixels produce a different amount of dark current under illumination. Finally, we discuss the implications that this has for dark frame image correction.
\end{abstract}

Keywords: digital images, image correction, dark current

\section{INTRODUCTION}

A major source of noise in charge-coupled device imagers is the generation of dark current. Even without the presence of light one finds that an image is not all black. White dots appear in the image and represent pixels with high signal caused by impurities located in the silicon wafer. The dark signal for all pixels is caused by thermal excitation of electrons from the valence into the conduction band of silicon. A basic understanding of dark current in a CCD can be gained by analyzing the dark current generated in the $\mathrm{p}-\mathrm{n}$ junction of a diode..$^{1-5}$ It has been shown that the non-uniformity in the dark count is caused by deep level impurities like $\mathrm{Ag}, \mathrm{Fe}$, or $\mathrm{Ni}^{6}{ }^{6-10}$ The fixed pattern of the dark count can be corrected by subtracting a so-called dark frame. A dark frame is generated by taking an image under the same condition as the light exposure (equal exposure time and temperature), except that the shutter remains closed. To decrease the dark current shot noise, multiple dark frames are taken and averaged to generate a so-called master frame. If one does not have the time to obtain a master dark frame for each imaging session, one can also calibrate the dark current of a CCD chip. After the imager is calibrated for a certain temperature and exposure time range one can compute a dark frame almost instantly. ${ }^{11}$

Every dark current subtraction assumes that the thermal generation of electrons is identical under illuminated and non-illuminated condition. Hence, one assumes that the dark current generation is not affected by the presence of light or by the electrons that have already been optically excited and collected in the well.

In this study we illuminated different CCDs with light to partially fill the well with electrons. We then compared the dark frame generated under the pre-illuminated condition to a conventional dark frame.

\section{EXPERIMENTAL SETUP}

For our study we used two different cameras: the SBIG ST-8XE with a KAF-1602E CCD sensor and the Meade Pictor 416XT with a KAF-0400 CCD sensor. Both CCD chips are buried-channel frontside-illuminated CCDs. The KAF$1602 \mathrm{E}$ is a $1530 \times 1020$ pixel array with an individual pixel size of $9 \mu \mathrm{m} \times 9 \mu \mathrm{m}$. The KAF-0400 has the same individual pixel size, but only $768 \times 512$ pixels. Both cameras are frequently used by astronomers.

We placed a LED operated with a constant current supply at a distance of about 10 to $20 \mathrm{~cm}$ from the camera so that the whole chip was illuminated approximately uniformly. In order to control the influx of light during an exposure an external shutter was mounted in front of the camera. The internal camera shutter was opened during all exposures. The

Sensors, Cameras, and Systems for Industrial/Scientific Applications IX, edited by Morley M. Blouke,

Erik Bodegom, Proc. of SPIE-IS\&T Electronic Imaging, SPIE Vol. 6816, 68160B

(c) 2008 SPIE-IS\&T $\cdot 0277-786 X / 08 / \$ 18$

SPIE-IS\&T/ Vol. 6816 68160B-1 
external shutter was opened for 100 to $800 \mathrm{~ms}$, at the beginning of the exposure. Only for the short time that the external shutter is opened did light reach the CCD. After the external shutter was closed, the remaining exposure was done in the "dark." The light level was controlled by varying the opening time of the external shutter, the current through the LED, and the distance between camera and light source. Immediately following each light exposure, a regular dark frame with the same exposure time was taken - again with the internal shutter open and the light on, but this time the external shutter remained closed throughout the whole exposure.

To measure the dark current over an integration time of $600 \mathrm{sec}$ with and without light, we took the following images.

1. $D L_{605}$ : Frame with the presence of light, $605 \mathrm{sec}$ exposure time, external shutter opened at the beginning of the exposure for 100 to $800 \mathrm{~ms}$

2. $D L_{5}$ : Frame with the presence of light, $5 \mathrm{sec}$ exposure time, external shutter opened at the beginning of the exposure for 100 to $800 \mathrm{~ms}$

3. $D_{605}:$ Dark frame, $605 \mathrm{sec}$, closed external shutter

4. $D_{5}$ : Dark frame, $5 \mathrm{sec}$, closed external shutter

The light exposure at the beginning is the same for the $D L_{605}$ and the $D L_{5}$ frames and is subtracted out by taking the difference of the two exposures. Therefore, the dark current, $D L_{600}$, accumulated during $600 \mathrm{sec}$ after the image was initially exposed to light is given as:

$$
D L_{600}=D L_{605}-D L_{5}
$$

The dark current, $D_{600}$, accumulated during $600 \mathrm{sec}$ while the camera was not exposed to light, is given as:

$$
D_{600}=D_{605}-D_{5}
$$

We will refer to this parameter as the normal dark count, since this is the count obtained in a regular dark frame.

To compare the dark count accumulated in $600 \mathrm{sec}$, one subtracts the dark count without light from the dark count with light:

$$
\Delta D=D_{600}-D L_{600}
$$

For each exposure type and time, 20 and 25 images were taken for the Pictor 416XT and ST-8XE, respectively. The data presented here are the average values of those images. All data is expressed in counts, the number of electrons for each count can be obtained from the gain of each camera. For the SBIG ST- 8 XE the gain is 2.3 counts/e- for the Meade Pictor 416XT the gain is 1.2 counts/e- The full well of ST-8XE and the 416XT was 43,500 counts and 85,000 counts, respectively. All data presented here is below the saturation level of the chip.

\section{RESULTS}

\section{SBIG ST-8XE}

One generally assumes that the dark current is independent of the light intensity. Under this assumption the dark current for the $D L_{600}$ and the $D_{600}$ frames should be the same and $\Delta D$ approximately zero. However, this is not true for all pixels and light intensities. Figure 1 shows the change in the dark count, $\Delta D$, as a function of the average count of the initial $5 \mathrm{sec}$ light exposure for six pixels. Independently of the initial illumination Pixels 4 and 5 do not show a

\begin{tabular}{|c|c|c|c|c|c|c|}
\hline Pixel & Group & $D_{600}$ in counts & $\begin{array}{c}\Delta D / D_{600} \\
\text { at } 2,000 \text { counts }\end{array}$ & $\begin{array}{c}\Delta D / D_{600} \\
\text { at } 8,500 \text { counts }\end{array}$ & $\begin{array}{c}\Delta D / D_{600} \\
\text { at } 16,000 \text { counts }\end{array}$ & $\begin{array}{c}\Delta D / D_{600} \\
\text { at } 26,000 \text { counts }\end{array}$ \\
\hline 1 & 1 & 1,139 & $60.4 \%$ & $83.8 \%$ & $82.1 \%$ & $85.6 \%$ \\
\hline 2 & 2 & 5,956 & $0.8 \%$ & $5.2 \%$ & $11.9 \%$ & $22.2 \%$ \\
\hline $3 a$ & $3 a$ & 2,716 & $7.5 \%$ & $24.0 \%$ & $39.0 \%$ & $51.7 \%$ \\
\hline $3 b$ & $3 b$ & 2,846 & $3.2 \%$ & $12.4 \%$ & $23.9 \%$ & $37.1 \%$ \\
\hline 4 & Major Peak & 184 & $-4 \%$ & $-5 \%$ & $10 \%$ & $-5 \%$ \\
\hline 5 & Minor Peak & 6,136 & $-0.6 \%$ & $-0.5 \%$ & $-0.8 \%$ & $0.0 \%$ \\
\hline
\end{tabular}
significant change in the dark count. For Pixels 2, 3a, and 3b the dark count decreases with increasing level of illumination.

Table 1. Normal dark count and change in dark count under illumination at the indicated counts. 


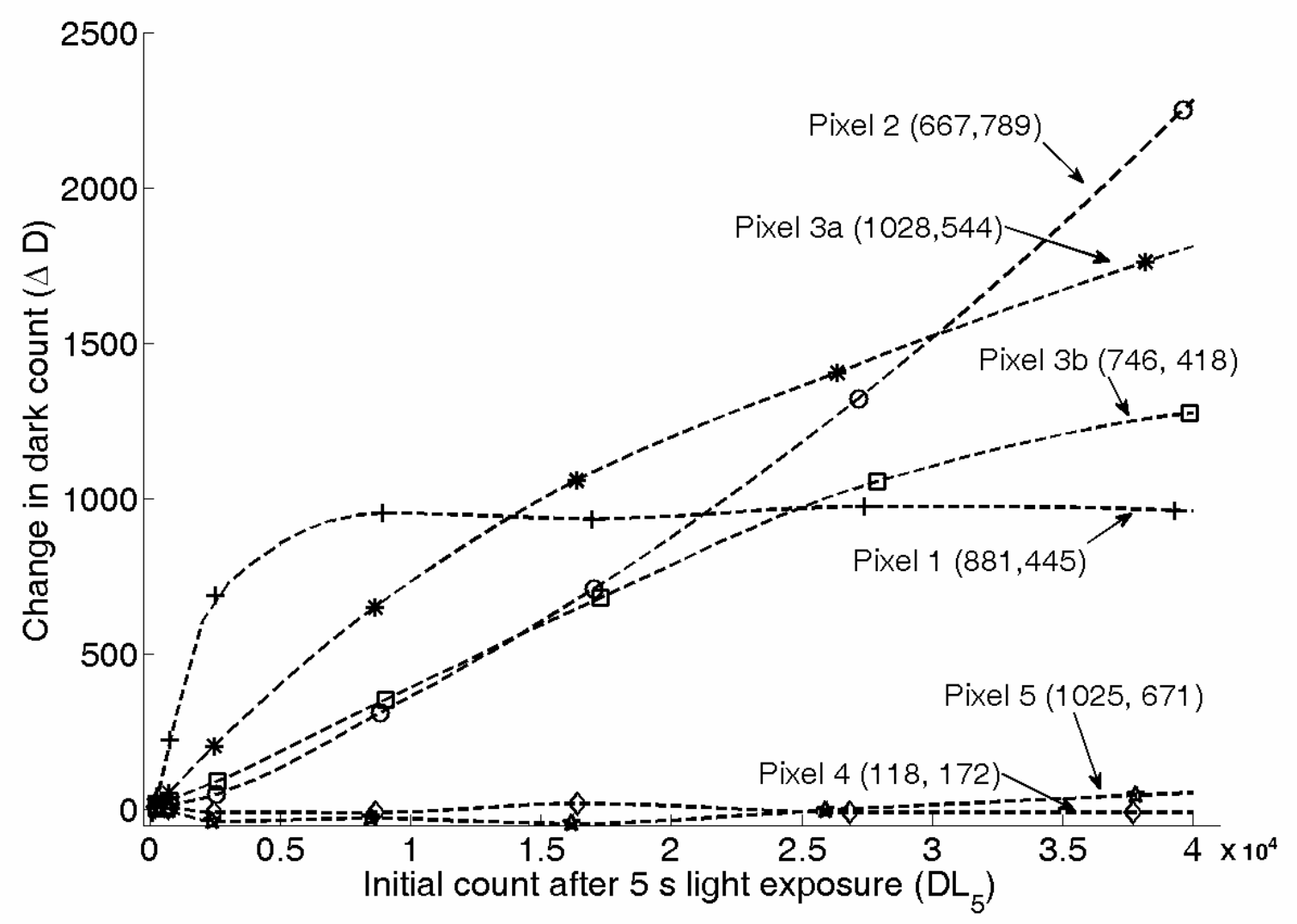

Figure 1: Loss in dark count vs. light level for 6 pixels for the ST-8XE. The dark count in the light exposures decreases with increasing light level. The numbers in the brackets are the $x$ and $y$ coordinates of the pixel.

The decrease in dark current slowly levels off for higher illumination levels. Pixel 1 also shows a decrease in dark count. Its decrease in dark count is roughly constant for initial light levels of 8,000 counts or more.

The normal dark count for the pixels in Fig. 1 can be seen in Table 1. At $288 \mathrm{~K}$ the average dark count across the chip without the presence of light, $D_{600}$, for a $600 \mathrm{sec}$ exposure is 397 counts. Except for Pixel 4, all pixels in Fig. 1 are hot pixels, that is the dark count is much larger than the average dark count of the image. As shown later each pixel represents a group of pixels with a distinct behavior under illumination.

A two-dimensional distribution of the loss in dark count, $\Delta D$, versus the dark count level, $D_{600}$, was created. The $\Delta D$ vs. $D_{600}$ plane was divided into intervals of size 50 counts by 50 counts and then, for each pixel according to its dark count values, the frequency for the corresponding interval is counted up by one. Figure 2 shows the resulting distributions for three different illumination levels. The shades of grey show how many pixels were in a specific interval. Due to the wide range of frequencies the grey scale is represented as the logarithm of the frequency.

All three panels in the figure show a characteristic form that gets more defined with increase of initial illumination level. For a specific dark count level, only very specific ranges of values for the change in dark count were observed. Based on the decrease in dark count we separated those pixels in different groups.

A large number of pixels have a small amount of dark current and the light exposure does not change this behavior. These pixels are seen in Fig. 2c with close to zero dark current and small or no change in the dark current (Main Peak). Approximately 1.5 million or 97 percent of all pixels behave this way (see also Table 2). Pixel 4 in Fig. 1 is one example of a pixel located in the Main Peak. Its normal dark count is about 184 counts and the change for all illumination levels is smaller than 20 counts. Pixel 5 in Fig. 1 is a pixel that is located in a second smaller peak of the distribution in Fig. 2. The approximately 16,600 pixels in this group have a large dark count, $D_{600}$, of about 6,000 counts and show no change under illumination. 
The greatest change in dark counts is exhibited by the pixels in Group 1. The change in dark count for those pixels is almost as high as their initial dark count. Hence, these pixels almost stopped generating dark current after being illuminated. One such pixel is Pixel 1 in Fig. 1. Under "normal" conditions, without the presence of light, its dark count at $288 \mathrm{~K}$ and $600 \mathrm{sec}$ exposure time is 1,139 counts. From Fig. 1 one observes that for an initial illumination level of roughly 8,000 counts or more, the decrease is almost 1,000 counts. This corresponds to only about 14 percent of the dark count without the presence of light (see Table 1).

In Fig. 2 a straight line can be drawn to fit all the pixels in Group 1. The slope of the straight line fit is larger than 0.9 for all three illumination levels depicted. As a result, pixels in Group 1 have a decrease in dark count of about 90 percent (e.g. $85.6 \%$ for Pixel 1 in Fig. 1) of the dark count. Hence, those pixels show almost no dark count when flashed with an initial illumination level of 8,000 counts or more (see Table 2).

Both Groups $3 a$ and $3 b$ show a decrease in dark level under illumination. Pixels $3 a$ and $3 b$ are representative of these groups. From Fig. 1, one can see that that level decreases with increasing illumination over the whole range of illumination levels. The percentage decrease of the dark count is smaller than for Group 1. The decrease of the dark level for those pixels for the different illumination levels can again be found from the slope of a linear fit through the pixels of these groups. Pixels in Group 3a have a decrease of about 25 percent at 8,600 counts illumination (Fig. 2a), 40 percent at 16,000 counts illumination (Fig. 2b), and 60 percent at 26,000 counts illumination (Fig. 2c). Hence, the dark current decrease is from 75 percent of the normal level at 8,600 count illumination to 40 percent of the normal level at 26,000 count illumination.

Group $3 \mathrm{~b}$ has the same characteristics as Group 3a, with the percentage decrease being slightly smaller. At 8,600 counts illumination the dark count is decreased by 15 percent, at 16,000 counts illumination it is decreased by 25 percent, and at 26,000 counts illumination it is decreased by 50 percent.

Finally, Group 2 is formed by pixels that have the same normal dark count as the pixels grouped in the minor peak. However, under illumination their dark current decreases. Like for Groups $3 \mathrm{a}$ and $3 \mathrm{~b}$, the dark count decreases with increasing illumination. Pixel 2 in Fig. 1 belongs to this group. The percentage change in dark count for all pixels in Fig. 1 can be seen in Table 1 .

A summary of the number of pixels in the individual groups and their change in dark count under different illumination levels can be seen in Table 2. Of the pixels outside these groups, 3,467 pixels have no change in dark count under illumination. Furthermore, there are a small number of pixels that have a dark count larger than in the minor peak and a slight decrease with illumination. We found that there is a significantly larger number of those pixels in the KAF-0400 chip and we will look at them in more detail in the next section.

\begin{tabular}{|l|c|c|c|c|c|}
\hline Group Name & \# Pixels & $\boldsymbol{D}_{600}$ in counts & $\begin{array}{c}\Delta \boldsymbol{D} \text { at } \\
\mathbf{8 , 5 0 0} \text { counts }\end{array}$ & $\begin{array}{c}\Delta \boldsymbol{D} \text { at } \\
\mathbf{1 6 , 0 0 0} \text { counts }\end{array}$ & $\begin{array}{c}\Delta \boldsymbol{D} \text { at } \\
\mathbf{2 6 , 0 0 0} \text { counts }\end{array}$ \\
\hline Major Peak & $1,519,003$ & 50 to 1,100 & -250 to 200 & -250 to 200 & -250 to 200 \\
\hline Minor Peak & 16,628 & 5,800 to 6,200 & -250 to 200 & -250 to 200 & -250 to 200 \\
\hline Group 1 & 3,638 & 1,000 to 6,000 & $90 \%$ of $D_{600}$ & $90 \%$ of $D_{600}$ & $90 \%$ of $D_{600}$ \\
\hline Group 2 & 2,260 & 5,500 to 6,600 & 250 to 3,250 & 250 to 3,250 & 250 to 3,250 \\
\hline Group 3a & 1,347 & 1,750 to 5,450 & $25 \%$ of $D_{600}$ & $40 \%$ of $D_{600}$ & $60 \%$ of $D_{600}$ \\
\hline Group 3b & 701 & 2,350 to 5,400 & $15 \%$ of $D_{600}$ & $25 \%$ of $D_{600}$ & $50 \%$ of $D_{600}$ \\
\hline Other & 17,023 & $\mathrm{xxx}$ & $\mathrm{xxx}$ & $\mathrm{xxx}$ & $\mathrm{xxx}$ \\
\hline
\end{tabular}

Table 2. Change in dark count for the different groups under different illumination levels. The number of pixels in each group was calculated using the constraints shown in Fig. 2 at 26,000 counts.

\section{Meade Pictor 416XT}

The second CCD investigated in this study was the Kodak KAF-0400 housed in the Meade Pictor 416XT. For the Pictor 416XT camera, data at $278 \mathrm{~K}$ was taken. The experimental setup and the analysis were done in the same way as for the SBIG camera. 

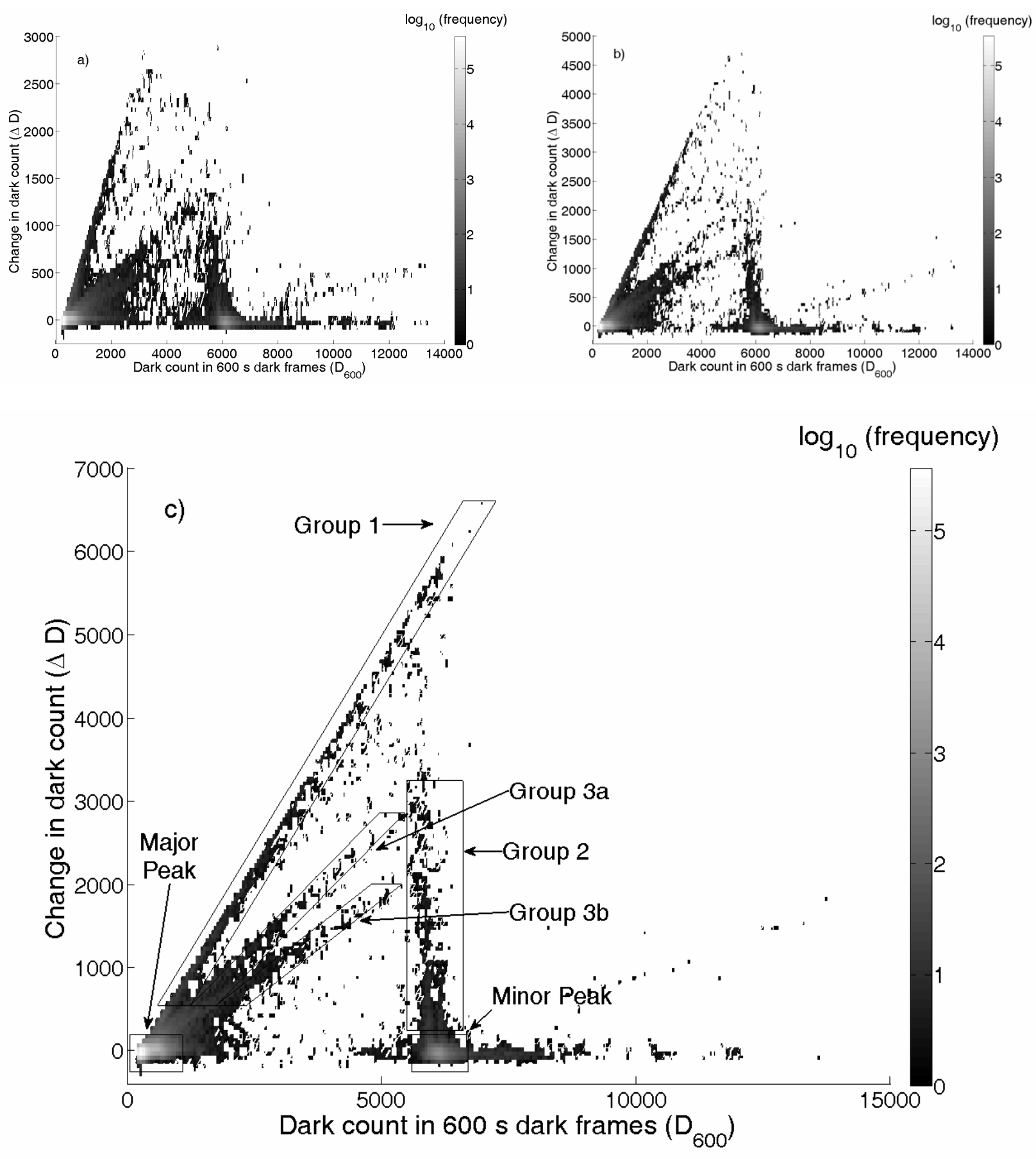

Fig. 2. Distribution of the change in dark count, $\triangle D$, at $288 \mathrm{~K}$ due to illumination for the SBIG ST-8XE for three different light levels. The average initial counts due to the light exposure were approximately: a) 8,500 counts, b) 16,000 counts, and c) 26,000 counts.

Figure 3 shows the distribution for the change in dark count for three different illumination levels. The images were flashed with light that caused an average count of approximately 8,500 (upper left panel), 16,000 (upper right panel) and 26,000 counts (lower panel). 
One finds many similarities and some differences in the characteristic for the two chips. For the Meade, instead of the two peaks, one finds another minor peak. The majority of pixels are regular pixels with very little dark current located in the major peak. Their average dark count at $278 \mathrm{~K}$ and $600 \mathrm{sec}$ exposure time is about 500 counts. There are two groups of pixels with a dark count of approximately 5,700 counts (Minor Peak 1) and 10,900 counts (Minor Peak 2), respectively. It is interesting to notice that pixels in all three peaks have a dark count of about 250 counts more when illuminated to light. The normal dark count of the pixels in Minor Peak 2 is about twice the dark count of the Minor Peak 1 pixels, making it likely that the same impurity occurs twice within that group.

Group 1a for the 416XT is analogous to Group 1 for the ST-8XE. A straight line drawn through the pixels of Group 1a has a slope of approximately 1 . Hence, pixels in this group generate almost no dark count when illuminated. Of course, to this one needs to add the global positive offset of about 250 counts. One can also see a number of pixels with a similar slope in the $D_{600}$ vs. $\Delta D$ graph emerge from the first minor peak. Because of this similarity we will call those pixels Group 1b. There are 1,376 pixels, less than 10 percent of Group 1a, in Group 1b (see Table 3). This is still a sizeable number, and one generally notices that there are a much larger number of pixels outside the Major Peak and Minor Peak groups for the Meade. Only about 80 percent of the pixels are in the Major Peak for the Meade camera, for the SBIG camera about 97 percent are in the Major Peak. One can explain pixels in Group 1b by assuming the presence of a Minor Peak 1 plus Group 1a type impurity in the same pixel.

Using the same reasoning for Group 2 one can conclude that Group 2b pixels contain a Minor Peak 1 plus a Group 2a type impurity. Again the number of pixels in Groups $2 \mathrm{a}$ and $2 \mathrm{~b}$ is much larger than the number of pixels in Group 2 of the SBIG camera. Only $0.15 \%$ percent of all pixels on the chip are in Group 2, while the percentage is much larger for the corresponding Group $2 \mathrm{a}$ and Group $2 \mathrm{~b}$ with $1.8 \%$ percent and $0.3 \%$ percent, respectively (see Table 2 and Table 3 ). Groups $3 \mathrm{a}$ and $3 \mathrm{~b}$ for the SBIG camera do not a have an equivalent in the Meade camera. Instead, there are a larger number of pixels (Group 3 with 1,751 pixels) with a normal dark count larger than the first minor peak and a significant loss in dark count. The slope of a linear fit through Group 3 pixels in Fig. 3 at 42,100 counts is given by about 0.3 . It appears likely that those pixels are the result of a Minor Peak 1 plus another secondary impurity. One can further notice from Fig. 2 that the SBIG camera seems to contain a similar type of pixel as well. However, just like for the rest of the chip, the number of pixels with impurities was smaller and we did not assign them in a separate group. Groups $2 b$ and 3 overlap and we counted 427 pixels twice as being both in Group 2b and in Group 3. 9,931 pixels are not in any of the groups, with 5,045 of those at the baseline with an increase in the dark count of approximately 250 counts.

\begin{tabular}{|c|r|c|c|c|}
\hline Group Name & \# Pixels & $\boldsymbol{D}_{\mathbf{6 0 0}}$ in counts & $\begin{array}{c}\Delta \boldsymbol{D} \text { at } \\
\mathbf{2 5 , 5 0 0} \text { counts }\end{array}$ & $\begin{array}{c}\Delta \boldsymbol{D} \text { at } \\
\mathbf{4 2 , 1 0 0} \text { counts }\end{array}$ \\
\hline Major Peak & 322,051 & 50 to 1600 & -700 to 100 & -700 to 100 \\
\hline Minor Peak 1 & 30,818 & 5,000 to 6,600 & -600 to 100 & -600 to 100 \\
\hline Minor Peak 2 & 1,550 & 10,100 to 11,800 & -450 to 100 & -450 to 100 \\
\hline Group 1a & 16,332 & 400 to 7,400 & $1 \times D_{600}-$ Off & $1 \times D_{600}-$ Off \\
\hline Group 1b & 1,376 & 6,200 to 11,700 & $1 \times\left(D_{600}-D_{M P I}\right)-$ Off & $1 \times\left(D_{600}-D_{M P I}\right)-$ Off \\
\hline Group 2a & 6,962 & 5,000 to 6,200 & 100 to 3,050 & 100 to 3,050 \\
\hline Group 2b & 1,250 & 10,100 to 11,500 & 100 to 3,300 & 100 to 3,300 \\
\hline Group 3 & 1,751 & 7,600 to 16,300 & $0.2 \times\left(D_{600}-D_{M P I}\right)-$ Off & $0.3 \times\left(D_{600}-D_{M P I}\right)-$ Off \\
\hline Group 2b and 3 & 427 & $\mathrm{xxx}$ & $\mathrm{xxx}$ & $\mathrm{xxx}$ \\
\hline Other & 9,931 & $\mathrm{xxx}$ & $\mathrm{xxx}$ & $\mathrm{xxx}$ \\
\hline
\end{tabular}

Table 3: Change in dark count for the different groups under different illumination levels. The parameter, Off=250 counts, represents the dark count decrease of a regular pixel under illumination. The dark count of a pixel in the first minor peak is given approximately as $D_{M P I}=5,700$ counts. The number of pixels in each group was calculated using the constraints shown in Fig. 3 at 42,100 counts. 

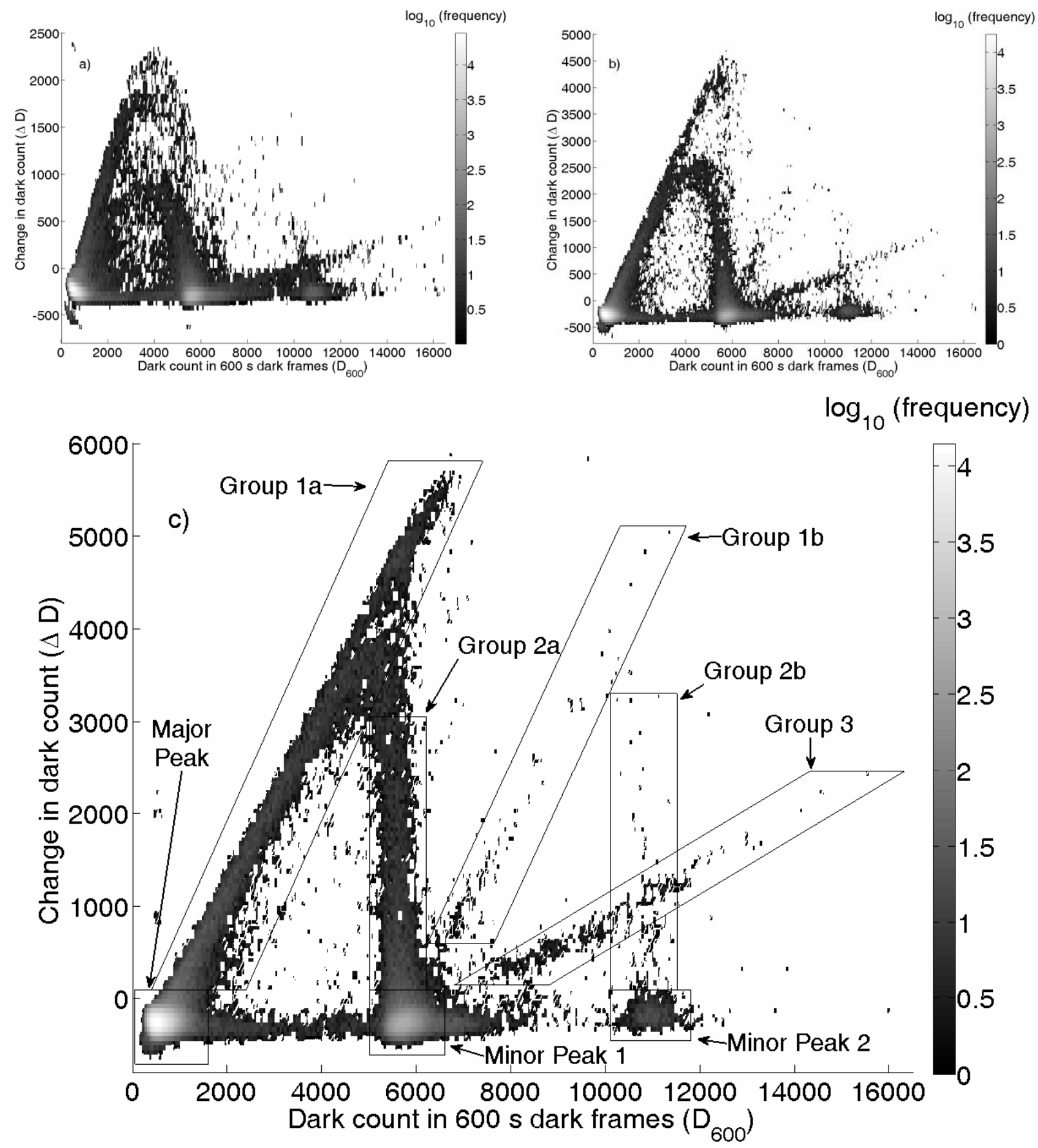

Fig. 3: Distribution of the change in dark count at $278 \mathrm{~K}$ due to illumination for the Pictor $416 X T$ for three different light levels. The average initial counts due to the light exposure were approximately: a) 12,500 counts, b) 25,500 counts, and c) 42,100 counts. 


\section{DISCUSSION}

We found that results are independent of the wavelength, $\lambda$, of the illuminating light and therefore independent of the location where photoelectrons are generated. The data for a red LED with $\lambda=650 \mathrm{~nm}$ and a penetration depth in silicon of $3 \mu \mathrm{m}$ yielded the same characteristics as a blue LED with $\lambda=430 \mathrm{~nm}$ and a penetration depth of only $300 \mathrm{~nm}$.

The distributions for the same light level at different temperatures were measured and the same characteristics were found at those temperatures as well. It was further noted that the pixels of all groups were found to be uniformly distributed over the whole area of the chip.

Most pixels for the SBIG camera fall in the two peaks and do not show a significant change. On the other hand, for larger intensities a majority of pixels for the Meade camera show an actual increase in dark count. Hence, a regular dark frame would predict a dark count which is too small. Our data indicate that this is a global phenomenon across the whole chip, resulting in a constant offset. What makes an accurate dark count correction more problematic is the fact that some pixels will show a decrease in dark count. Under large enough illumination, pixels in Group 1 almost stop generating dark electrons. Of course, in an actual exposure the photo-excitation of electrons due to the incoming light does not happen all at the beginning of the exposure. Assuming a constant intensity of light, pixels in Group 1 might generate dark current at a normal rate at the beginning of the exposure and then transforms to a non-dark count generating state toward the end of the exposure. Our analysis can help to predict how much dark count is generated during different stages of the exposure. This, however, assumes that the light intensity is constant and does not vary with time. If one does not have enough knowledge of possible changes in the light intensity during the exposure an accurate prediction of the dark count of pixels in Group 1 is virtually impossible.

The difficulties in correcting dark current accurately for pixels in Groups 2 and 3 are similar to those for pixels in Group 1. There are roughly 21,000 pixels, or $1.4 \%$ of the total pixels, for the SBIG camera ( $T=288 \mathrm{~K}, 600 \mathrm{sec}$ exposure time) where the dark current differs by more than 200 counts for an exposure with an initial illumination level of 26,000 counts. The same pixels would show a different dark count to a varying degree under all illumination condition. Ignoring the global increase of dark current under illumination, there are roughly 28,000 pixels, or $7.1 \%$ of the total pixels, in Groups 1, 2, and 3 that have a dark count that is strongly illumination level dependent for the Meade camera.

Finally, since dark current is strongly linked to impurities in the CCD our results can lead to a new understanding of the nature of those impurities. Impurities are generally characterized by the dark current they produce at a particular exposure time and temperature. Furthermore, by varying the temperature one can find the activation energy of a particular impurity. For example, the activation energy can be used to distinguish between diffusion current generated in the field-free region or depletion current from within the potential well. ${ }^{6}$ But the nature of impurities also differs by their behavior under illumination. A pixel in the Minor Peak and a Group 2 pixel show the same normal dark count. They would be characterized as being hot and containing one particular impurity. However, our data clearly shows that while they may have the same impurity, the nature of the defect in these pixels differs.

\section{CONCLUSION}

Dark current is generally assumed to be independent of the light level of the exposure. Differences between the actual number of dark electrons in the real exposure and a dark frame are generally assumed to be due to statistical noise. However, our study shows clearly that certain pixels show a systematic difference in their dark count under illumination.

The change in dark count $\Delta D$ in $600 \mathrm{sec}$ cannot assume any value - we found that only specific areas in the $\Delta D$ vs. $D_{600}$ plane were observed. We were able to group the behavior of pixels according to this discrete change. Pixels in one group may contain one specific type of impurity.

We hope this study will help contribute to a better understanding of the nature of dark current and an even further improvement of CCD image quality. More immediately the results help to accurately correct for dark current and in the computation of dark frames. 


\section{REFERENCES}

${ }^{1}$ A. S. Grove, "Physics and Technology of Semiconductor Devices," (John Wiley \& Sons, 1967)

${ }^{2}$ C. T. Sah, R. N. Noyce, and W. Shockley, "Carrier Generation and Recombination in p-n Junction and p-n Junction Characteristics," Proc. IRE, 45, 1228, 1957

${ }^{3}$ R. N. Hall, "Electron-Hole Recombination in Germanium," Phys. Rev. 87, 387, 1952

${ }^{4}$ W. Shockley and W. T. Read, "Statistics of the Recombination of Holes and Electrons," Phys. Rev. 87, 835, 1952

${ }^{5}$ S.M. Sze, "Physics of Semiconductor Devices," second edition (John Wiley \& Sons, 1981)

${ }^{6}$ R. Widenhorn, M. M. Blouke, A. Weber, A. Rest, and E. Bodegom, "Temperature dependence of dark current in a CCD," Proc. SPIE Int. Soc. Opt. Eng. 4669, 193, 2002

${ }^{7}$ R. D. McGrath, J. Doty, G. Lupino, G. Ricker, and J. Vallerga, “Counting of deep-level traps using a charge-coupled device”, IEEE Trans. Electron Devices, vol. ED-34, 2555, 1987

${ }^{8}$ W. C. McColgin, J. P. Lavine, J. Kyan, D. N. Nichols, and C. V. Stancampiano, "Dark current quantization in CCD image sensors," International Electron Device Meeting 1992, p. 113, 13-16 Dec., 1992

${ }^{9}$ W. C. McColgin, J. P. Lavine, and C. V. Stancampiano, "Probing metal defects in CCD image sensors," Mat. Res. Soc. Symp. Proc. 378, 713, 1995

${ }^{10}$ W. C. McColgin, J. P. Lavine, C. V. Stancampiano, and J. B. Russell, "Deep-level traps in CCD image sensors," Mat. Res. Soc. Symp. Proc. 510, 475, 1998

${ }^{11}$ R. Widenhorn, A. Rest, M. M. Blouke, R. L. Berry, E. Bodegom, “Computation of Dark Frames in Digital Imagers," SPIE 6501, 650103, 2007 